\title{
e-VLBI observations of the ULX at M33 - A status report
}

\author{
Alfonso Trejo*t \\ CRyA-UNAM, Morelia, Mexico \\ E-mail: a.trejo@crya.unam.mx \\ Zsolt Paragi \\ ${ }^{1}$ JIVE, Dwingeloo, Netherlands \\ ${ }^{2}$ MTA Research Group for Physical Geodesy and Geodynamics, Penc, Hungary \\ E-mail: zparagi@jive.nl
}

Kajal K. Ghosh

NASA/MFSC, USA

E-mail: kajal.k.ghosh@nasa.gov

\section{Sergei Fabrika}

Special Astrophysical Observatory, Niznhij Arkhyz, Russia

E-mail: fabrikaesao.ru

\section{Luis F. Rodríguez}

CRyA-UNAM, Morelia, Mexico

E-mail: 1 .rodriguez@crya.unam.mx

\section{Josep Martí}

Universidad de Jaén, Spain

E-mail: jmarti@ujaen.es

Some of the Ultraluminous X-Ray (ULX) sources have associated weak radio counterparts, but so far none of them has been detected on milliarcsecond scales. Observations with the very long baseline interferometry (VLBI) technique would be crucial to unveil the real nature of ULXs, however detecting sources so faint is very challenging. The ULX at the center of M33 is very close to us and hence a good target to study. Here we give a progress report, so far including 2 sessions of our e-EVN monitoring programme of this source.

Science and Technology of Long Baseline Real-Time Interferometry:

The 8th International e-VLBI Workshop, EXPReS09

June 22 - 262009

Madrid, Spain

*Speaker.

$\dagger$ e-VLBI developments in Europe are supported by the EC DG-INFSO funded Communication Network Developments project 'EXPReS', Contract No. 02662. The European VLBI Network is a joint facility of European, Chinese, South African and other radio astronomy institutes funded by their national research councils. 


\section{Ultraluminous X-ray Sources}

Ultraluminous X-Ray (ULX) sources, discovered in the 1980s, are pointlike non-nuclear objects that have $\mathrm{X}$-ray luminosities between $10^{39}$ and $10^{41} \mathrm{erg} \mathrm{s}^{-1}$ [1].2]. Considering the Eddington limit for an accreting object with isotropic X-ray emission, masses between 100 to $10000 \mathrm{M}_{\odot}$ were implied [2]. This indicated the presence of a new type of objects, Intermediate Mass Black Holes (IMBH). The IMBH interpretation has a number of difficulties however. For example, formation of these systems in sufficient numbers is problematic [3]. Alternatively, mildly beamed massive X-ray binaries could explain most of the ULX properties [3]. Should some of the most nearby ULXs host an IMBH, these could be revealed by their compact radio emission at detectable levels for sensitive VLBI arrays [4]. Milliarcsecond (mas) scale resolution is required to be able to distinguish the emission coming from or dominated by radio nebulae related to ULXs. This would be expected if ULXs are related to objects similar to the well known Galactic X-ray binary SS433, that is embedded in the W50 supernova remnant [5].

\section{M33 X-8}

M33 X-8 is an ULX with a 1-10 keV luminosity of $\sim 10^{39.5} \mathrm{erg} \mathrm{s}^{-1}$ [6]. The distance to the galaxy was considered to be $795 \mathrm{kpc}$ [7], though recent results have pointed out a value of approximately $950 \mathrm{kpc}[13$. The ULXs is found very close to the centre of M33. Detection of a 106 days periodicity in X-ray luminosity by ROSAT (Röntgensatellit) indicates that the emission is coming from a single source, rather than a number of stars [8]. On arcsecond scales, Trejo, Rodríguez \& Martí [9] detected faint emission from the M33 nucleus. At $20 \mathrm{~cm}(\theta \sim 1.5$ "), a source with $200 \mu \mathrm{Jy}$ was found, which is according to 2MASS (The 2-micron All Sky Survey) coincident with the M33 centre. At $3.6 \mathrm{~cm}(\theta \sim 0.4$ "), two unresolved sources were found with flux densities of 40 and $50 \mu \mathrm{Jy}$. The first one is coincident with the centre of the galaxy and the second one a bit off centre to the North.

In this work we are interested in the detection of the radio emission of the ULX source on the mas scale. To reach the required $5 \mu \mathrm{Jy} / \mathrm{beam}$ noise level, long integrations are required by current VLBI arrays. There is an advantage in splitting this integration time to several epochs of observation, since the radio source might be very variable. Our initial goal was to carry out 5-epoch e-EVN observations (including Arecibo in the array) at the highest possible data rate. So far two epochs have been scheduled. Using the e-VLBI technique instead of disk recording would let us carry out the full program on a shorter timescale (not limited to the regular EVN sessions), and as an additional advantage any flaring activity from the main target or other unrelated sources near the centre of M33 would be immediately noticed. With the combination of the data from various epochs one may detect the quiescent emission if the source is compact, or give better upper limit to its flux density.

\section{Observations and data processing}

The first observing session took place on 10 February 2009. The antennas in the array included Arecibo, Medicina, Onsala, Torun, Westerbork and Cambridge. Because of various problems, the 


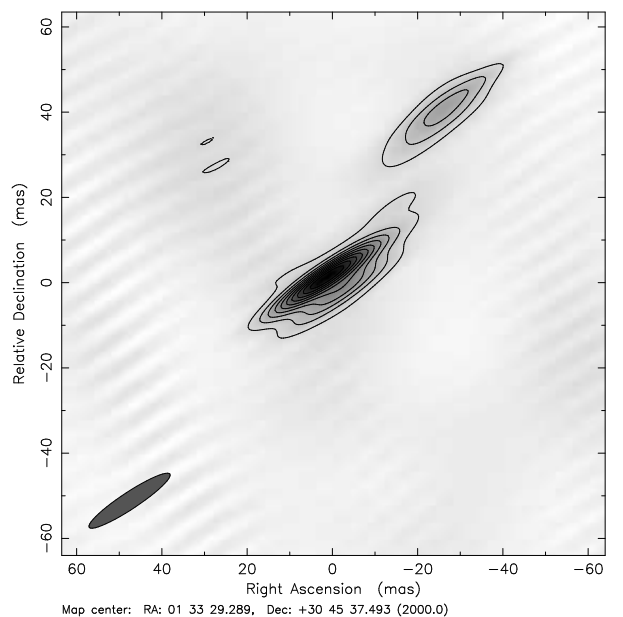

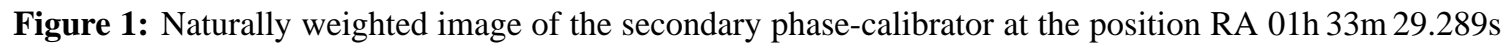
DEC 30d 45' 37"493 (J2000). The peak brightness is $8 \mathrm{mJy} / \mathrm{beam}$, the contour levels are multiples of 700 $\mu \mathrm{Jy} /$ beam. The beam is $22.7 \times 3.96$ mas oriented at a position angle of -56.6 degrees.

$76 \mathrm{~m}$ Lovell Telescope (at Jodrell Bank) and the 100m Effelsberg telescope could not participate (then a re-observation was granted). The observations were carried out at $1.6 \mathrm{GHz}$ in dual polarization using 2-bit sampling, at a total data rate per telescope of $512 \mathrm{Mbps}$; Arecibo was limited to $256 \mathrm{Mbps}$, and the MERLIN telescopes were limited to $128 \mathrm{Mbs}$. The total observing time was about 5.7 hours, with 2.8 hours on target. During the second epoch on 21-22 April 2009 Effelsberg and the Lovell Telescope were present, and in addition a MERLIN outstation was added, Darnhall. Out of the 8 hours total observing time 3.5 hours were spent on the target. The ULX was phase-referenced to J0137+3122 in both experiments, which is a bright and compact calibrator just 1 degree away. As a secondary candidate phase-calibrator, we included a $\sim 10 \mathrm{mJy}$ faint source in the phase-reference cycle, which was identified in a short e-EVN run in 2008. This secondary calibrator is only $\sim 7$ arcminutes away from the target. The sequence for the observations was J0137+3122 ( 1 min) - sec. cal. ( 1 min) - M33 X-8 ( $3 \mathrm{~min})-\mathrm{J} 0137+3122(\sim 1 \mathrm{~min})$, and so forth.

The AIPS astronomical package was used to process the data in the standard manner. We used the measured antenna gains and system temperatures for amplitude calibration. Then we ran the AIPS task FRING on J0137+3122 and applied the solutions to all sources. The data for the secondary calibrator were then self-calibrated and imaged. The obtained phase solutions from CALIB were transferred to the target. This step did not give a significant improvement of the result, indicating that there were no large residual phase errors left in the data after the first phase-reference step. In Fig. 1 we show a naturally weighted image of the secondary phase-calibrator, which shows a resolved structure.

\section{Preliminary results and discussion}

We still have no detection of the target in the first two observation epochs. Using natural weighting we could derive upper limits for both epochs. For the first one the measured rms noise 
was $24 \mu \mathrm{Jy} /$ beam and $13 \mu \mathrm{Jy} / \mathrm{beam}$ for the second epoch. At the end of the campaign we expect to get a much improved noise level from the combined data. The above noise level is still not sufficient to show if any of the components detected by [9] are compact. Furthermore, as we still do not have a detection, we can set a limit of 5 sigma level of $65 \mu \mathrm{Jy} / \mathrm{beam}$ for a variable source.

From the fundamental plane of black hole activity relation [10,11] one may give an approximate upper limit to the black hole mass from the observed X-ray luminosity, and the upper limit of radio flux density at $\mathrm{GHz}$ frequencies. For a $1000 \mathrm{M}_{\odot} \mathrm{IMBH}$ at the distance of M33 with $L_{X} \sim 10^{39}$ $\mathrm{erg} \mathrm{s}^{-1}$ one may expect to see a radio source at a few hundred $\mu \mathrm{Jy}$ level. The resolved nature of the $200 \mu \mathrm{Jy}$ source in the centre of M33 with the VLA [9] already makes this scenario unlikely. If the 40-50 $\mu \mathrm{Jy}$ VLA components remain undetected in the combined VLBI observations, this would be constrained further. With the data available at this time, our result is in agreement with the upper limit of $1500 \mathrm{M}_{\odot}$ from velocity dispersion measurements in the bulge of the galaxy [12].

\section{References}

[1] G. Fabbiano, X rays from normal galaxies, Ann. rev. Astron. Astrophys. 27 (1989) 87

[2] E. J. M. Colbert, R. F. Mushotzky, The Nature of Accreting Black Holes in Nearby Galaxy Nuclei, Astrophys. J. $\mathbf{5 1 9}$ (1999) 89

[3] A. R. King, M. B. Davies, M. J. Ward, G. Fabbiano, M. Elvis, Ultraluminous X-Ray Sources in External Galaxies, Astrophys J. 552 (2001) 109

[4] Z. Paragi, M. A. Garrett, A. D. Biggs, Observing ULXs with VLBI: the quest for intermediate-mass black holes, PoS(MQW6) (2006) 61

[5] S. Fabrika, A. Mescheryakov, Face-on SS 433 stars as a possible new type of extragalactic X-ray sources, IAU Symp. 205 (2001) 268

[6] L. Foschini, K. Ebisawa, T. Kawaguchi, N. Capelluti, P. Grandi, G. Malaguti, J. Rodríguez, T. J-L. Courvoisier, G. Di Cocco, L. C. Ho, G. G. C. Palumbo, The application of slim disk models to ULX: the case of M33 X-8 Adv. Space Res. 38(7) (2006) 1378

[7] S. van den Bergh, The stellar populations of M 33, Publ. Astron. Soc. Pac. 103 (1991) 609

[8] G. Dubus, P. Charles, K. S. Long, P. J. Hakala A 106 Day Period in the Nuclear Source X-8 in M33, Astrophys. J. 490 (1997) L47

[9] A. Trejo, L. F., Rodríguez, J. Martí, In Search of a Radio Counterpart for the Ultraluminous X-Ray Source at the Nucleus of M33, Rev. Mex. Astron. Astrophys. 40 (2004) 223

[10] A. Merloni, S. Heinz, T. Di Matteo, A Fundamental Plane of black hole activity, Mon. Not. Royal Astron. Soc. 345 (2003) 1057

[11] H. Falcke, E. Körding, S. Markoff, A scheme to unify low-power accreting black holes. Jet-dominated accretion flows and the radio/X-ray correlation, Astron. Astrophys. 414 (2004) 895

[12] K. Gebhardt, T. R. Lauer, J. Kormendy, J. Pinkney, G. A. Bower, R. Green, T. Gull, J. B. Hutchings, M. E. Kaiser, C. H. Nelson, D. Richstone, D. Weistrop, M33: A Galaxy with No Supermassive Black Hole, Astron. J. 122 (2001) 2469

[13] A. Z. Bonanos, K. Z. Stanek, R. P. Kudritzki, L. M. Macri, D. D. Sasselov, J. Kaluzny, P. B. Stetson, D. Bersier, F. Bresolin, T. Matheson, B. J. Bochejska, N. Przybilla, A. H. Szentgyorgyi, J. Tonry, G. Torres, The first direct distance determination to a detached eclipsing binary in M33, The Astrophysical Journal. 652 (2006) 313 